Serial assessment of the coagulation status of dogs with immune-mediated haemolytic anaemia using thromboelastography

Goggs, R.; Wiinberg, Bo; Kjelgaard-Hansen, Mads; Chan, D. L.

Published in:

The Veterinary Journal

DOI:

10.1016/..tvjl.2011.03.015

Publication date:

2012

Document version

Early version, also known as pre-print

Citation for published version (APA):

Goggs, R., Wiinberg, B., Kjelgaard-Hansen, M., \& Chan, D. L. (2012). Serial assessment of the coagulation status of dogs with immune-mediated haemolytic anaemia using thromboelastography. The Veterinary Journal, 191(3), 347-353. https://doi.org/10.1016/j.tvjl.2011.03.015 


\title{
Serial assessment of the coagulation status of dogs with immune-mediated haemolytic anaemia using thromboelastography
}

\author{
R. Goggs ${ }^{\mathrm{a}, *}$, B. Wiinberg ${ }^{\mathrm{b}}$, M. Kjelgaard-Hansen ${ }^{\mathrm{b}}$, D.L. Chan ${ }^{\mathrm{a}}$ \\ a Veterinary Clinical Sciences, Royal Veterinary College, Hawkshead Lane, North Mymms, Hertfordshire AL9 7TA, UK \\ ${ }^{\mathrm{b}}$ Department of Small Animal Clinical Sciences, Faculty of Life Sciences, University of Copenhagen, Gronnegaardsvej 3 St., DK-1870 Frederiksberg C, Denmark
}

\section{A R T I C L E I N F O}

Article history:

Accepted 16 March 2011

\section{Keywords:}

Hypercoagulability

Thromboelastography

Immune-mediated haemolytic anaemia

Prognosis

\begin{abstract}
A B S T R A C T
This study investigated the coagulation status of dogs with immune-mediated haemolytic anaemia (IMHA) over time. Thirty animals with primary IMHA were blood sampled on three occasions over a 5 day period and assays performed included prothrombin time, activated partial thromboplastin time, D-dimer and fibrinogen concentration, antithrombin activity and recalcified unactivated thromboelastography (TEG).

Based on TEG, dogs with IMHA were significantly hypercoagulable vs. controls $(P<0.001)$ and over the 5 day period, $3 / 4$ of the TEG parameters reflected increased clotting kinetics $(P \leqslant 0.02)$. The 30 day survival of these patients was $80 \%$ and, at hospital admission, the TEG maximum amplitude (MA) was significantly higher in survivors than non-survivors $(P=0.015)$. Each unit increase in MA was associated with an increased odds of 30 day survival of 1.13 (95\%; CI 1.02-1.25). Based on TEG, most dogs with IMHA were hypercoagulable on admission and their clotting kinetics increased with time. Relative hypocoagulability identified by TEG at initial assessment was found to be a negative prognostic indicator.
\end{abstract}

(c) 2011 Elsevier Ltd. All rights reserved.

\section{Introduction}

Various coagulation abnormalities have been documented in dogs with immune-mediated haemolytic anaemia (IMHA) including disseminated intravascular coagulation (DIC) and a hypercoagulable state (Mischke, 1998; Scott-Moncrieff et al., 2001; Carr et al., 2002). IMHA in dogs is associated particularly with pulmonary thromboembolism (Bunch et al., 1989; Klein et al., 1989; Johnson et al., 1999) but thrombosis has also been identified at other sites including the cranial vena cava (Palmer et al., 1998), portal vein (Van Winkle and Bruce, 1993), heart, spleen and kidney (McManus and Craig, 2001; Carr et al., 2002). There is an association between IMHA and a prothrombotic state and thromboembolism is linked with poor clinical outcome (Klag et al., 1993; Scott-Moncrieff et al., 2001; Carr et al., 2002). Mechanisms proposed for the generation of thromboembolism in IMHA include cytokine-induced monocyte and endothelial tissue factor expression (Hoffman, 2006), erythrocyte phosphatidylserine exposure (Ataga et al., 2007), procoagulant microparticle release (Horne et al., 2006) and the presence of anti-phospholipid antibodies (Pullarkat et al., 2002).

Until recently clinical recognition of hypercoagulability was difficult. Thromboelastography (TEG) is a patient-side, viscoelastic assessment of coagulation, which provides a graphical

\footnotetext{
* Corresponding author. Tel.: +441173311448.

E-mail address: rob.goggs@bristol.ac.uk (R. Goggs).
}

representation of clot formation over time (Kol and Borjesson, 2010; Wiinberg and Kristensen, 2010). TEG has been used to identify hypercoagulability in dogs with parvoviral enteritis (Otto et al., 2000), neoplasia (Kristensen et al., 2008), DIC (Wiinberg et al., 2008) and protein losing nephropathy (Hilling et al., 2009). The velocity curve ( $V_{\text {curve }}$ ) is a computed parametric derivative of a conventional TEG profile which enables quantification of the kinetics of clot propagation. The results of this analysis are similar to the parameters produced by DyCoDerivaAu software derived from Rotem tracings (Sorensen et al., 2003) and the TEG $V_{\text {curve }}$ has been described for plasma (Nielsen et al., 2004) and whole blood (Ellis et al., 2004).

A recent retrospective study reported the use of TEG to identify hypercoagulability in dogs with IMHA (Sinnott and Otto, 2009). Consistent with previous reports linking thrombosis with poor outcome in cases of IMHA, Sinnott and Otto (2009) hypothesised that dogs with IMHA would be hypercoagulable and that the resultant TEG profiles would be associated with a poor outcome. The study unexpectedly identified that no animals with a normal coagulation index survived, suggesting that dogs which were not hypercoagulable had a greater risk of death. This result appears counterintuitive. One interpretation is that animals with IMHA and normal coagulation indices are relatively hypocoagulable, potentially consistent with the presence of a consumptive coagulopathy. Thus a normal TEG profile in a dog with IMHA may not preclude the ongoing existence of such a coagulopathy. 
Although hypercoagulability has been identified in dogs with IMHA using TEG, the retrospective study of Sinnott and Otto (2009) was limited by a lack of standardisation of timing of TEG or concurrent analyses. Serial TEG was not performed and thus it remains unknown how treatment may alter these profiles. Corticosteroids, the mainstay of therapy for IMHA, are associated with an increased risk of thromboembolic complications (Van Winkle and Bruce, 1993; Palmer et al., 1998). Alterations in coagulation associated with corticosteroid administration have also been demonstrated with TEG, wherein prednisolone (at $1 \mathrm{mg} / \mathrm{kg}$ or $4 \mathrm{mg} / \mathrm{kg}$, once daily) significantly altered the TEG clot formation time $(K)$, clot formation angle $(\alpha)$, and maximum amplitude (MA) values consistent with a hypercoagulable profile (Rose et al., 2008). Whereas corticosteroids in normal dogs induce hypercoagulable TEG profiles, in animals with IMHA the immunosuppression should resolve the hypercoagulability. As a result, the effect of steroid therapy on the TEG profiles of dogs with IMHA is difficult to predict.

The objectives of this study were to investigate hypercoagulability in naturally occurring cases of IMHA in dogs using TEG and to assess changes in coagulation status over time in treated animals. Based on the results of Sinnott and Otto (2009), we hypothesised that dogs with IMHA would be hypercoagulable on initial assessment and those that did not demonstrate hypercoagulability would have a poorer survival rate. We also hypothesised that therapy would ameliorate the hypercoagulability associated with the disease.

\section{Materials and methods}

Study population

The study was approved by the Royal Veterinary College Ethics and Welfare Committee.

A cohort of 30 dogs with IMHA was selected based on sample size calculations (Uitenbroek, 1997; Otto et al., 2000), which suggested that this number would be required to detect two-way differences between groups in MA and reaction time $(R)$ with $80 \%$ power. Dogs admitted to the Queen Mother Hospital for Animals (QMHA), The Royal Veterinary College (RVC), London, between January 2008 and October 2009 and diagnosed with primary IMHA were considered eligible. The diagnostic criteria were: anaemia with a PCV $<37 \%$ and a positive in-saline agglutination test, Coombs' test or moderate-to-marked spherocytosis on a blood smear no evidence of underlying disease. Patients were not eligible if their platelet count was $<100,000 / \mu \mathrm{L}$ in order to exclude dogs with an Evans-like syndrome. To facilitate case recruitment dogs were selected provided they had not received corticosteroids for $>3$ days previously, in line with a previous study (Mitchell et al., 2009). Dogs receiving aspirin, clopidogrel, unfractionated or low-molecular weight heparin or warfarin were also excluded.

Signalment, clinical history, results of clinical examination and clinicopathological data were recorded on admission to the QMHA. To enable objective assessment of disease severity in the study population, the canine haemolytic anaemia objective score (CHAOS) was calculated (Whelan et al., 2006). Assessment of the inflammatory status of the dogs was based on published criteria (Okano et al., 2002). To exclude cases of secondary IMHA, all dogs underwent diagnostic evaluations at the discretion of the attending clinician including: abdominal ultrasound $(n=30)$; thoracic radiography $(n=29)$; urine culture $(n=27)$; urinalysis $(n=26)$; PCR screening for tick-borne disease where there was a history of travel outside of the UK $(n=5)$.

\section{Patient sampling}

Blood samples were collected by jugular venepuncture using a $21 \mathrm{G}$ needle and a $10 \mathrm{~mL}$ syringe and were immediately aliquoted into $1.1 \mathrm{~mL}$ or $1.3 \mathrm{~mL}$ non-vacuum, polypropylene tubes (Pediatric tube, International Scientific Supplies). Samples for complete blood counts (CBC) were collected into potassium EDTA tubes. Samples for serum biochemistry and C-reactive protein (CRP) were collected into gel separator tubes. For TEG, prothrombin time (PT), activated partial thromboplastin time (aPTT), antithrombin activity (AT), fibrinogen (Fg) and D-dimer determinations, blood was collected into tubes containing 3.2\% liquid sodium citrate. On days 0 and 3, CBCs, serum biochemistry, CRP, PT, aPTT, Fg, AT, D-dimers and TEG were carried out on $10 \mathrm{~mL}$ of blood. On day $5, \mathrm{CRP}, \mathrm{Fg}$, AT and TEG were performed using $5 \mathrm{~mL}$ of blood. Owing to financial constraints, a CBC was not performed on the day 5 samples. Blood PCV was measured at each time-point.
Sample analysis

CBCs and serum biochemistry were performed using automated analysers (CellDyn 2500, Abbott Laboratories and ILAB 600, Instrumentation Laboratory). Prothrombin and aPTTs were measured on citrated whole blood using a 'point-of-care' analyser (CoagDx, IDEXX). D-dimers were assayed by a commercial laboratory using a latex agglutination method (Helena Biosciences). C-reactive protein (CRP) was measured using an automated human turbidimetric assay validated for canine use (Kjelgaard-Hansen et al., 2003; Kjelgaard-Hansen, 2010) calibrated with purified canine CRP (LifeDiagnostics). Samples for Fg and AT were assayed by the Clauss method and chromogenic assay, respectively.

\section{Thromboelastography}

All samples were run on a single machine (TEG 5000 Haemostasis Analyser, Haemoscope Corporation) according to the manufacturer's instructions. To avoid time-dependent alterations in TEG parameters, samples were held at room temperature for 30 min prior to analysis (Bowbrick et al., 2000; Wiinberg et al., 2005). The assays were run for $90 \mathrm{~min}$. All TEG tracings were produced using a recalcified, unactivated assay. Thromboelastography was performed using a heparinase cup in dogs with in-dwelling IV catheters or in those receiving heparin $(n=73)$. Plain TEG cups were only used in dogs not receiving any form of heparin $(n=7)$.

Four variables were recorded from the TEG tracings: $R$; $K$; MA; and $\alpha$. Three variables were recorded from the corresponding $V_{\text {curve }}$ tracings: maximum rate of thrombus generation (MRTG); time to maximum rate of thrombus generation (TMRTG); and total thrombus generated (TG). Recalcified, unactivated TEG tracings were produced from a control population of 30 healthy dogs (HC), selected from the hospital blood donation programme. These dogs were considered healthy on the basis of: clinical examination; $\mathrm{CBC}$ and serum biochemistry profile; and absence of travel outside the UK.

\section{Statistical analysis}

Prior to test selection, data were assessed for normality. Although some variables were parametric, most were not and thus non-parametric tests were used throughout. Wilcoxon signed-rank tests were used to compare TEG results from dogs with IMHA with controls and to compare pre- and post-treatment TEG values. Calculation of the Spearman's rank correlation coefficient was used to assess relationships of continuous clinicopathological variables with TEG values. The Chi-square or Fisher's exact tests were used to compare categorical variables. Binary logistic regression was used to identify predictors of outcome. All analyses were conducted using commercial statistical software (SPSS 16 for Windows, SPSS Inc.) with $\alpha$ set at 0.05 . Where multiple comparisons were undertaken the $P$ value was adjusted using the rough false discovery rate technique (Benjamini and Yekutieli, 2001).

\section{Results}

\section{Study population characteristics}

Although 34 dogs were initially selected, four were excluded because of neoplasia $(n=2)$ and thrombocytopaenia $(n=2)$. The 30 remaining dogs had a mean age of $6.7 \pm 3.1$ years and were of 13 different breeds including: English Springer spaniel $(n=8)$; Cocker spaniel $(n=7)$; Labrador retriever $(n=2)$; and Staffordshire bull terrier $(n=2)$. There was one Bedlington terrier, Border collie, Dachshund, Golden retriever, Jack Russell terrier, Miniature schnauzer, Shih Tzu, Weimeraner and West Highland white terrier and two crossbred animals.

A high proportion of the study population was female (23/30, $76.7 \%$ ), of which 19 were neutered. Five of the seven males were neutered. The median duration of clinical signs prior to presentation was 3 days (1-7). Ten dogs had received no treatment prior to presentation. Thirteen animals had been given corticosteroids prior to presentation with some dogs receiving more than one drug: prednisolone $(n=9)$; dexamethasone $(n=5)$; and methylprednisolone $(n=1)$. The median duration of prior corticosteroid therapy was 1 day (1-3). Other treatments included clavulanatepotentiated amoxicillin $(n=6)$, vitamin K, sucralfate, ranitidine, azathioprine (each $n=3$ ), enrofloxacin $(n=2)$, metronidazole $(n=2)$, and opioids $(n=2)$. Three dogs received fresh whole blood prior to presentation and one animal had been given a haemoglobin-based oxygen carrying solution (HBOC) (Oxyglobin, Biopure). 
On presentation the mean heart rate was $133 \pm 23 \mathrm{bpm}$, the mean respiratory rate was $41 \pm 17 \mathrm{bpm}$ and the mean rectal temperature $38.6 \pm 0.6{ }^{\circ} \mathrm{C}$. All dogs had pale mucous membranes and 14 displayed clinical evidence of jaundice. Seven dogs had audible systolic murmurs (all $\leqslant 3 / 6$ ) and all 30 animals were in-saline agglutination positive. The initial clinicopathological findings are summarised in Table 1. Fifteen dogs were positive for systemic inflammatory response syndrome (SIRS) (Okano et al., 2002) and the median CHAOS score was 3 (2-6; maximum score 7). All the treatments administered at the QMHA are listed in Table 2.

\section{Clinical outcomes}

Twenty-five dogs were discharged alive and three died during hospitalisation: one due to uncontrollable haemolysis (on day 4); one due to secondary acute kidney failure (on day 5); and one due to suspected pulmonary thromboembolism (on day 2). A necropsy on the latter dog did not identify pulmonary emboli but did identify thrombi in the hepatic sinusoids, suggesting that this dog may have died of thromboembolic complications. Two dogs were euthanased during hospitalisation: one following acute clinical deterioration (on day 3 ) and another following failure to respond to therapy (on day 10). Neither of these cases was available for necropsy.

Of the 25 dogs discharged from the hospital, 24 were alive 30 days post-admission and 17 were still alive 6 months postadmission (equivalent to a 58.6\% 6 month survival rate). One dog was 'lost' to follow-up. Although median CHAOS values for non-survivors were higher than for survivors indicating more severe disease, the difference was not significant at time-of-discharge from hospital or at 30 days post-admission. Neither the SIRS status (Okano et al., 2002), nor any of the other clinicopathological variables tested were predictive of clinical outcome at either of these time-points.

\section{Thromboelastography}

At admission, none of the TEG variables were significantly different between dogs treated and not treated with corticosteroids.
At hospital admission, compared to controls, dogs with IMHA had significantly shorter $K$ times, greater $\alpha$ angles and greater MA values (all $P<0.001$ ) consistent with a hypercoagulable state. The TEG variables changed significantly following initiation of therapy (Table 3 ). From admission to day 3 there were significant decreases in $R(P=0.023)$ and $K(P=0.006)$ and a significant increase in $\alpha(P=0.012)$. At day 5 , all TEG variables were significantly different from those at admission: $R$ and $K$ were significantly decreased $(P \leqslant 0.001)$; MA was significantly decreased $(P=0.021)$; and $\alpha$ was significantly increased $(P=0.003)$ (Fig. 1$)$.

At day 5 all of the TEG values remained significantly different from those of the controls $(P \leqslant 0.002)$ with tracings having a more hypercoagulable profile over time, except for the MA values which decreased. Based on $V_{\text {curve }}$ analysis, the MRTG increased significantly between admission and day $5(P=0.012)$ and the TMRTG decreased significantly over the same time period $(P=0.001)$. There was a significant decrease in TG between admission and day $5(P=0.031)$ (Table 3$)$.

A number of variables correlated with the TEG derived MA values at admission. Maximum amplitude positively correlated with Fg concentration $(r=0.790, P<0.001 ; n=28)$ and with AT activity $(r=0.532, P=0.004 ; n=28)$. Maximum amplitude negatively correlated with PCV $(r=-0.451, P=0.012 ; n=30)$. Maximum amplitude did not significantly correlate with platelet count or with D-dimer concentration. Of the TEG variables, only MA was predictive of outcome. The MA at time of admission was significantly higher in survivors vs. non-survivors at discharge $(P=0.016)$ and at 30 days post-admission $(P=0.015)$ (Fig. 2$)$. Logistic regression analysis suggested each unit increase in MA was associated with increased odds of 30 day survival of 1.13 (95\%; CI $1.02-1.25)$.

\section{Other clinicopathological findings}

There were no significant correlations between $R, K$ or $\alpha$ and clotting times although aPTT values negatively correlated with MA at time of admission $(r=-0.529, P=0.0026 ; n=28)$. In all 28

Table 1

Summary clinicopathological data from 30 dogs with immune-mediated haemolytic anaemia at the time of admission to The Queen Mother Hospital for Animals. Reference intervals are those determined at and used in the hospital with the exception of the thromboelastography variables which represent the values from the 30 healthy controls (italicised).

\begin{tabular}{|c|c|c|c|c|c|c|}
\hline Variable & Median & Range & $n$ & $n$ below interval & $n$ above interval & Reference interval \\
\hline Albumin $(\mathrm{g} / \mathrm{L})$ & 29.7 & $18-39.5$ & 29 & 12 & 1 & $28-39$ \\
\hline $\operatorname{ALT}(\mu / \mathrm{L})$ & 53 & $10-1671$ & 29 & 1 & 12 & $13-88$ \\
\hline $\operatorname{ALP}(\mu / L)$ & 228 & $88-2185$ & 29 & 0 & 13 & 19-285 \\
\hline Bilirubin $(\mu \mathrm{mol} / \mathrm{L})$ & 41 & $2-1259$ & 29 & - & 28 & $0-2$ \\
\hline Neutrophils $\left(\times 10^{9} / \mathrm{L}\right)$ & 21.5 & $6.2-57.8$ & 30 & 0 & 24 & $3-11.5$ \\
\hline Band neutrophils $\left(\times 10^{9} / \mathrm{L}\right)$ & 0.9 & $0-5.9$ & 30 & - & 19 & $0-0.3$ \\
\hline Monocytes $\left(\times 10^{9} / \mathrm{L}\right)$ & 2.7 & $0.4-12.2$ & 30 & 0 & 24 & $0.15-1.5$ \\
\hline Platelet count $\left(\times 10^{9} / \mathrm{L}\right)$ & 192 & $112-400$ & 30 & 4 & 0 & $150-450$ \\
\hline Absolute reticulocyte count (cells $/ \mu \mathrm{L}$ ) & 155,400 & $1820-576,978$ & 19 & - & - & - \\
\hline PCV (\%) & 14.5 & $7-35$ & 30 & 30 & 0 & $37-55$ \\
\hline$R(\min )$ & 14.0 & $6.5-24.8$ & 30 & 5 & 2 & $9.1-20.3$ \\
\hline$K(\min )$ & 4.2 & $1.6-11.1$ & 30 & 16 & 0 & $4.3-13.1$ \\
\hline$\alpha$ angle $\left(^{\circ}\right)$ & 45.3 & $20.3-71.8$ & 30 & 0 & 19 & $17.0-42.4$ \\
\hline $\mathrm{MA}(\mathrm{mm})$ & 69.7 & $44.8-85.4$ & 30 & 0 & 28 & $33.5-49.0$ \\
\hline MRTG (mm/min) & 6.7 & $1.9-20.3$ & 30 & 0 & 21 & $1.5-4.7$ \\
\hline TMRTG (min) & 18.5 & $8.8-36.0$ & 30 & 5 & 3 & $11.6-27.6$ \\
\hline $\mathrm{TG}(\mathrm{mm} / \mathrm{min})$ & 840.7 & $548.6-1025.4$ & 30 & 0 & 28 & $396-595$ \\
\hline D-dimers (ng/mL) & $500-1000$ & $<250->2000$ & 30 & - & 23 & $<250$ \\
\hline C-reactive protein $(\mathrm{mg} / \mathrm{L})$ & 137 & $30.4-438.6$ & 27 & - & 27 & $0-8.9$ \\
\hline Fibrinogen $(\mu \mathrm{mol} / \mathrm{L})$ & 35.9 & $15-71.4$ & 28 & 0 & 28 & $4.3-14.1$ \\
\hline AT $(\%)$ & 94 & $63-125$ & 28 & 2 & 0 & $65-145$ \\
\hline PT (s) & 14 & $9-19$ & 29 & 3 & 1 & $12-17$ \\
\hline aPTT (s) & 106.5 & $92-150$ & 28 & 0 & 18 & $71-102$ \\
\hline
\end{tabular}

$\alpha$, clot formation angle; ALT, alanine aminotransferase; ALP, alkaline phosphatase; aPTT, activated partial thromboplastin time; AT, antithrombin activity; $K$, clot formation time; MA, maximum amplitude; MRTG, maximum rate of thrombus generation; PCV, packed cell volume; PT, prothrombin time; $R$, reaction time; TG, total thrombus generated; TMRTG, time to maximal rate of thrombus generation. 
Table 2

Summary of treatments administered to the 30 dogs with immune-mediated haemolytic anaemia following admission to The Queen Mother Hospital for Animals. All dogs were given corticosteroids, typically both dexamethasone and prednisolone. Where more than one corticosteroid was administered, this was given sequentially and not contemporaneously. Data is presented as median (range) unless otherwise specified. Types of therapeutic intervention are grouped together in the table.

\begin{tabular}{|c|c|c|c|}
\hline Treatment & $n$ & Median dose (range) & Frequency \\
\hline Prednisolone (generic) & 26 & $2 \mathrm{mg} / \mathrm{kg}(1.67-2.2)$ & Twice daily \\
\hline Dexamethasone (Dexadreson, Intervet) & 24 & $0.3 \mathrm{mg} / \mathrm{kg}(0.25-0.5)$ & Once daily \\
\hline Azathioprine (Imuran, GlaxoSmithKline) & 16 & $2 \mathrm{mg} / \mathrm{kg}(1.44-2.2)$ & Once daily \\
\hline Mycophenolate mofetil (Cellcept, Roche) & 6 & $16.3 \mathrm{mg} / \mathrm{kg}(10-20)$ & Once daily \\
\hline Cyclosporine (Atopica, Novartis) & 3 & $5 \mathrm{mg} / \mathrm{kg}(5-10)$ & Twice daily \\
\hline Human immunoglobulin (Kiovig, Baxter) & 2 & $0.49 \mathrm{~g} / \mathrm{kg}$ and $0.67 \mathrm{~g} / \mathrm{kg}$ & Single dose \\
\hline Packed red blood cells & 26 & $22.6 \mathrm{~mL} / \mathrm{kg}(6.6-46.7)$ & - \\
\hline Fresh whole blood & 2 & $18.7 \mathrm{~mL} / \mathrm{kg}$ and $27 \mathrm{~mL} / \mathrm{kg}$ & Single doses \\
\hline Haemoglobin-based oxygen carrying (HBOC) solution (Oxyglobin, Biopure) & 2 & $16.9 \mathrm{~mL} / \mathrm{kg}$ and $375 \mathrm{~mL} / \mathrm{kg}$ & Single doses \\
\hline Fresh frozen plasma & 1 & $25 \mathrm{~mL} / \mathrm{kg}$ & Single dose \\
\hline Omeprazole (Losec, AstraZeneca) & 12 & All $1 \mathrm{mg} / \mathrm{kg}$ & Once daily \\
\hline Sucralfate (Antepsin, Chugai Pharma) & 2 & Both $0.5 \mathrm{~g}$ & Three times daily \\
\hline Aspirin (generic) & 29 & All $0.5 \mathrm{mg} / \mathrm{kg}$ & Once daily \\
\hline Clopidogrel (Plavix, SanofiAventis) & 1 & $1.1 \mathrm{mg} / \mathrm{kg}$ & Once daily \\
\hline Dalteparin (Fragmin, Pfizer) & 3 & All $150 \mu / \mathrm{kg}$ & Three times daily \\
\hline Unfractionated heparin (generic) & 1 & $200 \mu / \mathrm{kg}$ & Three times daily \\
\hline
\end{tabular}

Table 3

Summary coagulation test data from the 30 dogs with immune-mediated haemolytic anaemia at time of admission (day 0 ), on day 3 and on day 5 . $P$ values correspond to comparisons between admission (day 0 ) and day 5 apart from those values indicated by ${ }^{*}$ where the $P$ value corresponds to the comparison between admission (day 0 ) and day 3 . Bold font signifies significance at $P<0.05$ or, where applicable, after adjustment for multiple comparisons.

\begin{tabular}{|c|c|c|c|c|c|c|c|}
\hline \multirow[t]{2}{*}{ Variable (unit) } & \multicolumn{2}{|l|}{ On admission (Day 0) } & \multicolumn{2}{|l|}{ Day 3} & \multicolumn{2}{|l|}{ Day 5} & \multirow[t]{2}{*}{$P$} \\
\hline & Median (range) & $n$ & Median (range) & $n$ & Median (range) & $n$ & \\
\hline$R(\min )$ & $14.0(6.5-24.8)$ & 30 & $10.9(5.7-22.9)$ & 27 & $9.8(4-22.9)$ & 23 & $<0.001$ \\
\hline$K(\min )$ & $4.2(1.6-11.1)$ & 30 & $2.8(1.3-13.1)$ & 27 & $2.4(0.9-10.4)$ & 23 & 0.001 \\
\hline$\alpha\left(^{\circ}\right)$ & $45.3(20.3-71.8)$ & 30 & $54.0(17.7-71.2)$ & 27 & $60.2(21.8-76.3)$ & 23 & 0.003 \\
\hline $\mathrm{MA}(\mathrm{mm})$ & $69.7(44.8-85.4)$ & 30 & $67.1(47.8-82.5)$ & 27 & $66.7(49.5-81.8)$ & 23 & 0.021 \\
\hline MRTG (mm/min) & $6.7(1.9-20.3)$ & 30 & $8.9(2.2-19.0)$ & 27 & $11.5(2.7-23.2)$ & 23 & 0.012 \\
\hline TMRTG (min) & $18.5(8.8-36.0)$ & 30 & $13.8(8.7-37.9)$ & 27 & $12.3(4.9-34.8)$ & 23 & 0.001 \\
\hline $\mathrm{TG}(\mathrm{mm} / \mathrm{min})$ & $840.7(548.6-1025.4)$ & 30 & $803.4(580.2-988.5)$ & 27 & $806.9(587.2-979.9)$ & 23 & 0.031 \\
\hline Platelet count $\left(\times 10^{9} / \mathrm{L}\right)$ & $200(110-400)$ & 30 & $165(50-400)$ & 27 & - & - & $0.882^{*}$ \\
\hline Fibrinogen $(\mu \mathrm{mol} / \mathrm{L})$ & $35.9(15-71.4)$ & 28 & $26.7(10.7-76.9)$ & 24 & $25(6.9-43.7)$ & 11 & 0.066 \\
\hline Antithrombin (\% normal) & $94(63-125)$ & 28 & $72.5(46-100)$ & 24 & $69(48-88)$ & 11 & 0.012 \\
\hline C-reactive protein $(\mathrm{mg} / \mathrm{L})$ & $137(30.4-438.6)$ & 27 & $57.7(7-336.6)$ & 26 & $38(2.9-156.7)$ & 23 & $<0.001$ \\
\hline D-dimers $(\mathrm{ng} / \mathrm{mL})$ & $500-1000(<250->2000)$ & 30 & $1000-2000(<250->2000)$ & 27 & - & - & $0.089^{*}$ \\
\hline PT $(s)$ & $14(9-19)$ & 29 & $12.5(11-18)$ & 24 & - & - & $0.633^{*}$ \\
\hline aPTT $(s)$ & $106.5(92-150)$ & 28 & $100(80-122)$ & 23 & - & - & 0.007* \\
\hline PCV (\%) & $14.5(7-35)$ & 30 & $22(4-34)$ & 27 & $24(15-39)$ & 23 & 0.003 \\
\hline
\end{tabular}

$\alpha$, clot formation angle; aPTT, activated partial thromboplastin time; AT, antithrombin activity; $K$, clot formation time; MA, maximum amplitude; MRTG, maximum rate of thrombus generation; PCV, packed cell volume; PT, prothrombin time; $R$, reaction time; TG, total thrombus generated; TMRTG, time to maximal rate of thrombus generation.

animals in which Fg concentrations were measured, the value was above normal at admission. Median Fg concentrations decreased between time of admission and day 5 but this was not significant $(P=0.066)$ and at day $5,91 \%$ of dogs still had abnormally high $\mathrm{Fg}$ concentrations. At admission, CRP concentrations were abnormally high in all 27 dogs in which this parameter was measured. There was a positive correlation between Fg and CRP concentrations at time of admission $(r=0.576, P<0.001 ; n=27)$. By day 5 , median CRP concentrations reduced significantly $(P<0.001)$, although $21 /$ 23 values remained abnormal. At time of admission, three dogs had AT activities below the reference interval. AT activity reduced significantly between admission and day $5(P=0.012)$ such that by day $5,36 \%$ of dogs had subnormal AT activities.

\section{Discussion}

Of the clinicopathological and TEG parameters assessed, only MA at initial presentation was significantly associated with outcome in this prospective study of dogs with IMHA. The results are consistent with those of previous studies (Sinnott and Otto,
2009; Flint et al., 2010). Based on the TEG parameters, dogs with IMHA exhibited evidence of hypercoagulability with significantly shorter $K$ times, greater $\alpha$ and higher MAs than controls. This indicates that affected dogs have both increased clotting kinetic activity and achieve higher total clot strengths. Consistent with our findings, a small study of 11 dogs identified significantly shorter $K$ times, larger $\alpha$ angles and higher MA values in dogs with IMHA at time of admission to hospital (Fenty et al., 2011). The cause of this hypercoagulability is likely multifactorial including hyperfibrinogenaemia, increased contact pathway activation, platelet hyperreactivity (Weiss and Brazzell, 2006), haemolysis (Bauer et al., 2010) and the effects of immunosuppressive drugs.

Within the first 5 days of therapy, $R, K$ and $\alpha$ changed significantly in a manner consistent with increased clotting kinetics. The changes in the $V_{\text {curve }}$ parameters, MRTG and TMRTG are also consistent with an increase in the rate of clot propagation over this time period. Indeed, since the conventional TEG values are nonparametric, clot propagation expressed as MRTG may provide a more accurate assessment of clot kinetics than either MA or $\alpha$ (Ellis et al., 2004). Decreased $K$ and increased $\alpha$ may reflect contempora- 

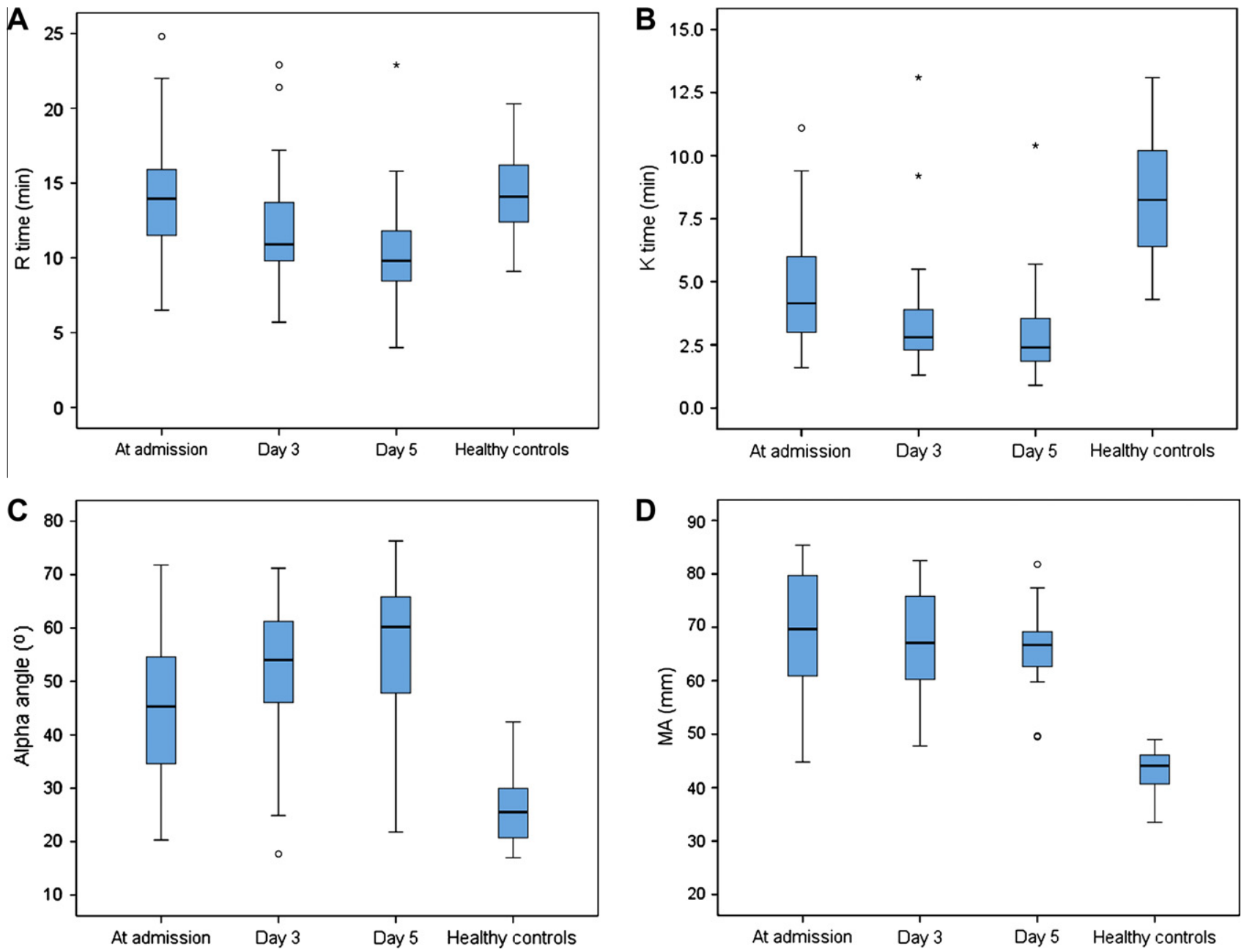

Fig. 1. 'Box and whisker' plots of thromboelastography variables from dogs with immune-mediated haemolytic anaemia at admission, and at post-admission days 3 and 5 , compared to controls. The middle lines represent the median, the boxes represent inter-quartile ranges and whiskers the 95th percentiles. Values outside the 95th and 99th percentiles are represented as open circles and by ${ }^{*}$, respectively. (A-D) illustrate reaction time $(R)$, clot formation time $(K)$, clot formation angle $(\alpha)$ and maximum amplitude (MA) values, respectively.

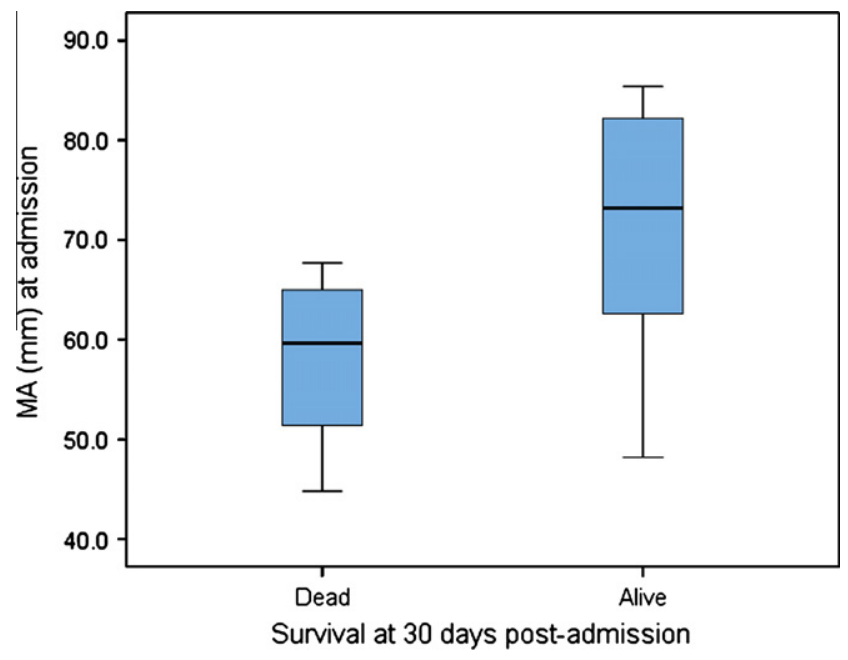

Fig. 2. 'Box and whisker' plots comparing the thromboelastography maximum amplitude (MA) values at hospital admission of surviving dogs, with those of animals that did not survive to 30 days post-admission. The MA values of nonsurvivors were significantly lower $(P=0.015)$. neous decreases in AT due to consumption secondary to increased thrombin production. The concomitant decreases in $R$ and aPTT suggest increased contact pathway activation may be the source of thrombin activation.

There are several potential explanations for this increase in contact pathway activation. Platelet activation, known to be increased in IMHA (Weiss and Brazzell, 2006), may occur through leucocyte interactions (Li, 2008), or nitric-oxide depletion (Rother et al., 2005), contributing to contact pathway activation through increased tenase complex assembly. Ongoing haemolysis may also contribute through continued generation of phosphatidylserinebearing erythrocyte-derived microparticles (Horne et al., 2006). Corticosteroid therapy may also be partly responsible (Jacoby et al., 2001) since hypercortisolism in both humans and dogs is associated with significantly increased concentrations of factors VIII, IX and XII (Chandler, 1995; Van Zaane et al., 2009). Increased thrombin generation, by promoting the development of DIC, might be one of the mechanisms responsible for the development of organ dysfunction or of thrombosis in dogs with IMHA.

The decrease in MA and in the $V_{\text {curve }}$ parameter TG between admission and day 5 were the only TEG-derived changes consistent with decreased hypercoagulability. However at all time-points the MA and TG values indicated significant hypercoagulability compared to controls and the decreases in MA and TG, while statisti- 
cally significant, were small in magnitude compared with other TEG variables. Maximum amplitude is positively correlated with platelet number, platelet function, $\mathrm{Fg}$ and thrombin generation (Chandler, 1995) and is negatively correlated with haematocrit (Zuckerman et al., 1981).

Identification of hypercoagulability in dogs with IMHA using TEG is hampered by the anaemia because of the inverse relationship between haematocrit and MA (Iselin et al., 2001; Spiezia et al., 2008). However, if the hypercoagulability documented by MA was solely due to alterations in haematocrit, it might be expected that the PCV would be associated with outcome: this was not the case. In the dogs in the current study, the MA was more closely correlated with $\mathrm{Fg}$ concentration $(r=0.790)$ and was only moderately correlated with PCV $(r=-0.451)$. Thus it is likely that some, but not all, of the increase in MA was due to anaemia. The use of euvolaemic, haemo-depleted controls produced by phlebotomy with autologous platelet-rich plasma transfusions might facilitate the evaluation of the specific contribution of haematocrit to the MA abnormalities observed.

The hyperfibrinogenaemia observed in the dogs in our study was likely associated with the inflammatory response since Fg and CRP concentrations were positively correlated. The strong correlation between Fg and MA in this study suggests the Fg concentration and hence the inflammatory response contributes significantly to the pathogenesis of the hypercoagulable syndrome in IMHA. Since platelet count has been reported to directly correlate with MA, the lack of correlation between platelet count and MA in our study is interesting. This may have been due to our exclusion criteria, which, by excluding patients with platelet counts $<100,000 / \mu \mathrm{L}$, may have reduced the platelet count variability in the study population.

By day 5, the PCV significantly increased while Fg concentrations decreased although not significantly. The platelet count also decreased between admission and day 3 although not significantly. Nevertheless, these variables may all have contributed to the decrease in MA over time. If the decrease in MA values were associated with a consumptive coagulopathy we would have expected to find that the animals with low MA values had higher concentrations of D-dimers and lower ATs. We might also have expected the platelet count, Fg concentration and AT to fall over time due to consumption. Indeed, MA directly correlated with AT and the platelet count, Fg concentration and AT also all decreased over time. The median D-dimer value increased between admission and day 3 although MA and D-dimer concentrations were not significantly correlated. As such, the correlations between the TEG variables and the other measures of coagulation are consistent with the hypothesis proposed by Sinnott and Otto (2009) that a consumptive coagulopathy is responsible for the poorer outcome in patients with lower MA values on presentation.

This study is not without its limitations. Due to financial constraints not all tests were run at every time-point: D-dimer concentrations and platelet counts were not determined at day 5 . Although the recalcified, unactivated TEG assay may have higher inherent variability than the tissue factor or kaolin activated assays (Bauer et al., 2009), these assays were either not available to us or the work reporting their use had not been published at the commencement of our study. Similarly, we did not run complete factor analyses or assay fibrinolytic proteins, which might have provided additional information regarding the cause of the hypercoagulability.

Approximately half of the affected dogs in this study had received corticosteroids prior to hospital admission. No statistically significant differences were identified in the TEG parameters at presentation between dogs that had received such prior treatment and those that had not. However, given that corticosteroid administration to dogs is known to affect these parameters (Rose et al., 2008), we cannot exclude the possibility that this may have biased our results. In our study population, there was marked heterogeneity in the treatments administered. This lack of uniformity potentially introduces confounding factors which make it more difficult to relate cause and effect. Despite this heterogeneity however, the observation that MA was predictive of outcome appears significant and is consistent with previous findings (Sinnott and Otto, 2009; Flint et al., 2010).

Although we propose that relative hypocoagulability in IMHA is associated with a poor prognosis due to a consumptive coagulopathy, the lack of association between a 'hypercoagulable' TEG tracing and outcome remains puzzling. Does such a tracing truly represent an in vivo hypercoagulable state? A longitudinal study evaluating the association between TEG parameters and the risk of thrombosis will be required to address this question. Such a study will necessitate the involvement of multiple veterinary hospitals in order to provide sufficient statistical power given the inevitable heterogeneity in the disease population.

\section{Conclusions}

The results of this study indicate that TEG MA values at hospital admission are predictive of clinical outcome in dogs with IMHA. All the animals had TEG profiles consistent with hypercoagulability during hospitalisation, a state which was likely multifactorial in origin. Our data support the previously suggested hypothesis that consumptive coagulopathy is responsible for the poorer outcome in dogs with lower MA values on presentation. Thromboelastographic tracings thus appear to be predictive of outcome and as such should be considered integral to the assessment of dogs with IMHA.

\section{Conflict of interest statement}

None of the authors of this paper has a financial or personal relationship with other people or organisations that could inappropriately influence or bias the content of this paper.

\section{Acknowledgement}

This study was funded in part by The Petplan Charitable Trust (Project number 07-34) and The Waltham Foundation.

\section{References}

Ataga, K.I., Cappellini, M.D., Rachmilewitz, E.A., 2007. Beta-thalassaemia and sickle cell anaemia as paradigms of hypercoagulability. British Journal of Haematology 139, 3-13.

Bauer, N., Eralp, O., Moritz, A., 2009. Establishment of reference intervals for kaolinactivated thromboelastography in dogs including an assessment of the effects of sex and anticoagulant use. Journal of Veterinary Diagnostic Investigation 21, 641-648.

Bauer, N., Eralp, O., Moritz, A., 2010. Effect of hemolysis on canine kaolin-activated thromboelastography values and ADVIA 2120 platelet activation indices. Veterinary Clinical Pathology 39, 180-189.

Benjamini, Y., Yekutieli, D., 2001. The control of the false discovery rate in multiple testing under dependency. Annals of Statistics 29, 1165-1188.

Bowbrick, V.A., Mikhailidis, D.P., Stansby, G., 2000. The use of citrated whole blood in thromboelastography. Anesthesia and Analgesia 90, 1086-1088.

Bunch, S.E., Metcalf, M.R., Crane, S.W., Cullen, J.M., 1989. Idiopathic pleural effusion and pulmonary thromboembolism in a dog with autoimmune hemolytic anemia. Journal of the American Veterinary Medical Association 195, 17481753.

Carr, A.P., Panciera, D.L., Kidd, L., 2002. Prognostic factors for mortality and thromboembolism in canine immune-mediated hemolytic anemia: A retrospective study of 72 dogs. Journal of Veterinary Internal Medicine 16, 504-519.

Chandler, W.L., 1995. The thromboelastography and the thromboelastograph technique. Seminars in Thrombosis and Hemostasis 21, 1-6.

Ellis, T.C., Nielsen, V.G., Marques, M.B., Kirklin, J.C., 2004. Thromboelastographic measures of clot propagation: A comparison of alpha with the maximum rate of thrombus generation. Blood Coagulation and Fibrinolysis 18, 45-48. 
Fenty, R.K., Delaforcade, A.M., Shaw, S.E., O'Toole, T.E., 2011. Identification of hypercoagulability in dogs with primary immune-mediated hemolytic anemia by means of thromboelastography. Journal of the American Veterinary Medical Association 238, 463-467.

Flint, A., Abrams-Ogg, S., Kruth, S., Bersenas, A., Wood, R., 2010. Thromboelastography in dogs with immune-mediated hemolytic anemia treat with prednisone, azathioprine and low-dose aspirin. Journal of Veterinary Internal Medicine 24, 681.

Hilling, K.M., Labato, M.A., de Laforcade, A.M., Shaw, S., 2009. Documentation of hypercoagulability in protein-losing nephropathy via thromboelastography in 10 dogs. Journal of Veterinary Internal Medicine 23, 690.

Hoffman, P.C., 2006. Immune hemolytic anemia - selected topics, hematology. American Society of Hematology Education Program 1, 13-18.

Horne III, M.K., Cullinane, A.M., Merryman, P.K., Hoddeson, E.K., 2006. The effect of red blood cells on thrombin generation. British Journal of Haematology 133, 403-408.

Iselin, B.M., Willimann, P.F., Seifert, B., Casutt, M., Bombeli, T., Zalunardo, M.P., Pasch, T., Spahn, D.R., 2001. Isolated reduction of haematocrit does not compromise in vitro blood coagulation. British Journal of Anaesthesia 87, 246-249.

Jacoby, R.C., Owings, J.T., Ortega, T., Gosselin, R., Feldman, E.C., 2001. Biochemical basis for the hypercoagulable state seen in Cushing syndrome. Archives of Surgery 136, 1003-1006.

Johnson, L.R., Lappin, M.R., Baker, D.C., 1999. Pulmonary thromboembolism in 29 dogs: 1985-1995. Journal of Veterinary Internal Medicine 13, 338-345.

Kjelgaard-Hansen, M., 2010. Comments on measurement of C-reactive protein in dogs. Veterinary Clinical Pathology 39, 402-404.

Kjelgaard-Hansen, M., Jensen, A.L., Kristensen, A.T., 2003. Evaluation of a commercially available human C-reactive protein (CRP) turbidometric immunoassay for determination of canine serum CRP concentration. Veterinary Clinical Pathology 32, 81-87.

Klag, A.R., Giger, U., Shofer, F.S., 1993. Idiopathic immune-mediated hemolytic anemia in dogs: 42 cases (1986-1990). Journal of the American Veterinary Medical Association 202, 783-788.

Klein, M.K., Dow, S.W., Rosychuk, R.A., 1989. Pulmonary thromboembolism associated with immune-mediated hemolytic anemia in dogs: Ten cases (1982-1987). Journal of the American Veterinary Medical Association 195, 246-250.

Kol, A., Borjesson, D.L., 2010. Application of thromboelastography/ thromboelastometry to veterinary medicine. Veterinary Clinical Pathology 39, 405-416.

Kristensen, A.T., Wiinberg, B., Jessen, L.R., Andreasen, E., Jensen, A.L., 2008. Evaluation of human recombinant tissue factor-activated thromboelastography in 49 dogs with neoplasia. Journal of Veterinary Internal Medicine 22, 140-147.

Li, N., 2008. Platelet-lymphocyte cross-talk. Journal of Leukocyte Biology 83, 10691078.

McManus, P.M., Craig, L.E., 2001. Correlation between leukocytosis and necropsy findings in dogs with immune-mediated hemolytic anemia: 34 cases (19941999). Journal of the American Veterinary Medical Association 218, 1308-1313.

Mischke, V.R., 1998. Hemostatic disorders as a complication of autoimmune hemolytic anemia in dogs. Deutsche Tierärztliche Wochenschrift 105, 13-16.

Mitchell, K.D., Kruth, S.A., Wood, R.D., Jefferson, B., 2009. Serum acute phase protein concentrations in dogs with autoimmune hemolytic anemia. Journal of Veterinary Internal Medicine 23, 585-591.

Nielsen, V.G., Lyerly III, R.T., Gurley, W.Q., 2004. The effect of dilution on plasma coagulation kinetics determined by thromboelastographic is dependent on antithrombin activity and mode of activation. Anesthesia and Analgesia 99, 1587-1592.

Okano, S., Yoshida, M., Fukushima, U., Higuchi, S., Takase, K., Hagio, M., 2002. Usefulness of systemic inflammatory response syndrome criteria as an index for prognosis judgement. Veterinary Record 150, 245-246.
Otto, C.M., Rieser, T.M., Brooks, M.B., Russell, M.W., 2000. Evidence of hypercoagulability in dogs with parvoviral enteritis. Journal of the American Veterinary Medicine Association 217, 1500-1504.

Palmer, K.G., King, L.G., Van Winkle, T.J., 1998. Clinical manifestations and associated disease syndromes in dogs with cranial venal cava thrombosis: 17 cases (1989-1996). Journal of the American Veterinary Medical Association 213, 220-224.

Pullarkat, V., Ngo, M., Iqbal, S., Espina, B., Liebman, H.A., 2002. Detection of lupus anticoagulant identifies patients with autoimmune haemolytic anaemia at increased risk for venous thromboembolism. British Journal of Haematology 118, 1166-1169.

Rose, L., Bedard, C., Dunn, M., 2008. Effects of prednisone administration on thromboelastography parameters in healthy beagles. Journal of Veterinary Internal Medicine 22, 739.

Rother, R.P., Bell, L., Hillmen, P., Gladwin, M.T., 2005. The clinical sequelae of intravascular hemolysis and extracellular plasma hemoglobin - A novel mechanism of human disease. Journal of the American Medical Association 293, 1653-1662.

Scott-Moncrieff, J.C., Treadwell, N.G., McCullough, S.M., Brooks, M.B., 2001. Hemostatic abnormalities in dogs with primary immune-mediated hemolytic anemia. Journal of the American Animal Hospital Association 37, 220-227.

Sinnott, V.B., Otto, C.M., 2009. Use of thromboelastography in dogs with immunemediated hemolytic anemia: 39 cases (2000-2008). Journal of Veterinary Emergency and Critical Care 19, 484-488.

Sorensen, B., Johansen, P., Christiansen, K., Woelke, M., Ingerslev, J., 2003. Whole blood coagulation thromboelastographic profiles employing minimal tissue factor activation. Journal of Thrombosis and Haemostasis 1, 551-558.

Spiezia, L., Radu, C., Marchioro, P., Bertini, D., Rossetto, V., Castelli, M., Pagnan, A., Sørensen, B., Simioni, P., 2008. Peculiar whole blood rotation thromboelastometry (Rotem) profile in 40 sideropenic anaemia patients. Thrombosis and Haemostasis 100, 1106-1110.

Uitenbroek, D.G., 1997. Sample Size, SISA. http://home.clara.net/sisa/samsize.htm (accessed 12 January 2007).

Van Winkle, T.J., Bruce, E., 1993. Thrombosis of the portal vein in eleven dogs. Veterinary Pathology 30, 28-35.

Van Zaane, B., Nur, E., Squizzato, A., Dekkers, O.M., Twickler, M.T., Fliers, E., Gerdes, V.E., Büller, H.R., Brandjes, D.P., 2009. Hypercoagulable state in Cushing's syndrome: A systematic review. Journal of Clinical Endocrinology and Metabolism 94, 2743-2750.

Weiss, D.J., Brazzell, J.L., 2006. Detection of activated platelets in dogs with primary immune-mediated hemolytic anemia. Journal of Veterinary Internal Medicine 20, 682-686.

Whelan, M.F., Rozanski, E.A., O’Toole, T.E., Crawford, S., Holm, J., Cotter, S.M., 2006. Use of the canine hemolytic anemia objective score (CHAOS) to predict survival in dogs with immune mediated hemolytic anemia. Journal of Veterinary Internal Medicine 20, 714-715.

Wiinberg, B., Kristensen, A.T., 2010. Thromboelastography in veterinary medicine. Seminars in Thrombosis and Hemostasis 36, 747-756.

Wiinberg, B., Jensen, A.L., Rojkjaer, R., Johansson, P., Kjelgaard-Hansen, M., Kristensen, A.T., 2005. Validation of human recombinant tissue factor activated thromboelastography on citrated whole blood from clinically healthy dogs. Veterinary Clinical Pathology 34, 389-393.

Wiinberg, B., Jensen, A.L., Johansson, P.I., Rozanski, E., Tranholm, M., Kristensen, A.T., 2008. Thromboelastographic evaluation of hemostatic function in dogs with disseminated intravascular coagulation. Journal of Veterinary Internal Medicine 22, 357-365.

Zuckerman, L., Cohen, E., Vagher, J.P., Woodward, E., Caprini, J.A., 1981. Comparison of thromboelastograph with common coagulation tests. Thrombosis and Haemostasis 46, 752-756. 